\title{
Altered fronto-limbic activity in children and adolescents with familial high risk for schizophrenia
}

\author{
Sarah J. Hart ${ }^{a, b},{ }^{,}$, Joshua Bizzella,b, Mary Agnes McMahon ${ }^{b}$, Hongbin Gua $^{a}$, Diana O. \\ Perkins $^{a}$, and Aysenil Belger ${ }^{a, b}$ \\ aDepartment of Psychiatry, University of North Carolina at Chapel Hill School of Medicine, Chapel \\ Hill, NC, USA \\ bDuke-UNC Brain Imaging and Analysis Center, Duke University Medical Center, Durham, NC, \\ USA
}

\begin{abstract}
Early symptoms of schizophrenia tend to emerge during adolescence, which is a critical period for development of executive and emotional processing. While individuals with familial high risk (FHR) for schizophrenia may show cognitive and emotional changes, the neural mechanisms underlying the development of these changes remain unclear. The goal of this study was to identify functional differences in fronto-striato-limbic regions in children with FHR. Functional MRI data was collected from 21 children with a first-degree family member with schizophrenia and 21 controls without FHR. Participants performed an emotional oddball task requiring both selective attention and suppression of task-irrelevant emotional information. During selective attention, the group with FHR showed enhanced activation in inferior frontal gyrus and caudate, with decreases in middle frontal gyrus and insular activation. The FHR group also showed greater age-related recruitment of anterior cingulate, temporal and occipital cortical areas during selective attention. During emotional processing, the FHR group showed decreased anterior cingulate activation, with decreased age-related recruitment of inferior frontal, parietal and occipital areas. The results suggest that FHR for schizophrenia may be associated with abnormal hyperactivation and hypoactivation of the neural circuitry engaged during executive and emotional processing and with age-related changes in neural recruitment during adolescence.
\end{abstract}

\section{Keywords}

Adolescence; Selective attention; Emotion; Prefrontal cortex

\section{Introduction}

Schizophrenia is widely considered to be a genetically-mediated neurodevelopmental disorder (Kety et al., 1971; Weinberger, 1987) characterized by deficits in both executive and emotional processing (Nuechterlein and Dawson, 1984; Nuechterlein et al., 1994). In individuals with familial high risk for developing schizophrenia by having an affected first-

\footnotetext{
(C) 2013 Elsevier Ireland Ltd. All rights reserved.

*Correspondence: Sarah J. Hart, Ph.D., Department of Psychiatry, CB \# 7160, University of North Carolina at Chapel Hill, Chapel Hill, NC 27599, sjhart@email.unc.edu, Office: 919-843-3153, Fax: 919-966-8004.

Publisher's Disclaimer: This is a PDF file of an unedited manuscript that has been accepted for publication. As a service to our customers we are providing this early version of the manuscript. The manuscript will undergo copyediting, typesetting, and review of the resulting proof before it is published in its final citable form. Please note that during the production process errors may be discovered which could affect the content, and all legal disclaimers that apply to the journal pertain.
} 
degree family member, neurocognitive deficits have been demonstrated across cognitive and social-emotional domains (Keshavan et al., 2010). This suggests the possibility that at-risk individuals may show early changes in brain function associated with executive and emotional processing preceding the onset of potential symptoms. These changes have been argued to be candidate vulnerability markers for schizophrenia (Gottesman and Gould, 2003; Gur et al., 2007a), warranting further research examining the developmental trajectory of executive and emotional functions in individuals with familial risk.

Individuals who have a first-degree family member with schizophrenia have an 8- to 12-fold increase in risk for developing the disorder (Faraone et al., 1999), with a rate of conversion to psychosis at approximately 10 percent (Glatt et al., 2006). In individuals with familial risk, cognitive deficits are among the first changes to appear, and are the strongest predictors of future development of the disorder (Cornblatt and Keilp, 1994), with deficits typically intermediate between patients and controls (Whalley et al., 2005a). These functional changes in at-risk individuals have been hypothesized to reflect inefficiency in cortical processing, where decreased dorsolateral prefrontal activity is seen when task demands exceed individual capacity (Jacobson et al., 2010), and increased compensatory activity may be needed to attain comparable levels of task performance to controls (Manoach et al., 1999). Several studies have demonstrated that unaffected adult relatives of individuals with schizophrenia show aberrant activity in the dorsolateral prefrontal cortex and other interconnected regions across a range of many different types of cognitive paradigms (Pearlson and Calhoun, 2009), including working memory (Meyer-Lindenberg and Weinberger, 2006), sentence completion (Whalley et al., 2005b) and memory encoding (Bonner-Jackson et al., 2007). Additionally, abnormal fronto-striatal interactions have been found to be related to exaggerated dopamine transmission in schizophrenia (MeyerLindenberg et al., 2002). Both individuals with schizophrenia and unaffected relatives have shown abnormalities in stratial function related to increased task demand, potentially reflecting reduced cortical control over the striatum (Vink et al., 2006).

Social-emotional changes have also been found in unaffected relatives, with impairments in emotional discrimination ability (Gur et al., 2007b). Additionally, volumetric reductions in the amygdala-hippocampal complex have been detected in unaffected young children of individuals with schizophrenia (Keshavan et al., 2002). More recently, functional imaging studies of individuals with familial risk have demonstrated abnormal interactions between the amygdala and prefrontal cortex during affective processing (Diwadkar et al., 2012) and during the resting state (Tian et al., 2011), suggesting a potential genetic influence on the function of these neural circuits.

While both executive and emotional systems have been found to be abnormally engaged in adults with schizophrenia and familial risk, it is unclear how early the changes in each system emerge during brain development. Adolescence may be a particularly vulnerable developmental period for schizophrenia, as it is associated with the emergence of symptoms that have been hypothesized to be related to an increase in pruning of synaptic connections (Feinberg, 1982). Structural MRI studies in adolescents with childhood-onset schizophrenia and their siblings have shown progressive gray matter loss in parietal cortex followed by frontal and temporal areas, in a pattern has been interpreted to be an exaggeration of the normal trajectory of gray matter loss in adolescence (Gogtay et al., 2003; Gogtay, 2008). However, it is unclear whether adolescents at risk for schizophrenia show a similar exaggeration of normal age-related changes in functional activity. The normal maturation pattern of neural circuits in adolescence is associated with development of more specialized and integrated activity within fronto-striate circuits (Rubia et al., 2006), with greater activation relating to improved task performance (Luna et al., 2001; Luna et al., 2010). The adolescent pattern has been interpreted to reflect compensation for immaturities in the 
circuits, as greater effort must be expended to achieve adult-like performance (Luna et al., 2010). Adolescence is also associated with less effective prefrontal modulation of amygdala activity compared to adults, reflecting immaturity in emotional regulation (Hare et al., 2008). Characterizing how the development of these processes changes in individuals at risk for schizophrenia will be critical for our understanding of the pathophysiology of the disorder.

In order to tap into the neural circuitry supporting executive and emotional processing, we chose to use an emotional oddball paradigm during functional MRI (fMRI) scanning to determine how familial risk for schizophrenia and age-related changes during adolescence may impact their function. The standard oddball paradigm has been frequently used to tap into fronto-striate circuitry by measuring selective attention processes allocated to taskrelevant target stimuli, and has elicited significant differences in both patients with schizophrenia (Kiehl and Liddle, 2001) and individuals with familial risk (Bramon et al., 2005) compared to controls. Modification of the oddball task to include task-irrelevant emotional stimuli has been shown to elicit activation changes in individuals with schizophrenia during processing of aversive stimuli (Dichter et al., 2010).

The goal of the present study was therefore to identify functional changes in executive and emotional processing that accompany cortical maturation in adolescents with familial risk for developing schizophrenia. Here we present cross-sectional data comparing the baseline assessments of our groups with and without familial risk. It should be noted that data acquisition for the longitudinal phase of the study is still ongoing. Given the hypothesis of inefficient cortical processing, we expected that during processing of task-relevant target stimuli and task-irrelevant emotional distracters, the group with familial risk for schizophrenia would show hyperactivation in fronto-striate circuitry compared to controls without familial risk. We also expected that the two groups would show differential changes in age-related activation patterns within networks supporting executive and emotional processing compared to controls, with greater development of hyperactivation in older adolescents due to increased ability to compensate for task demands.

\section{Methods}

\subsection{Participants}

We tested 42 age-, gender-, and ethnicity-matched subjects for this study, including 21 children (ages 9-18) with familial high risk for developing schizophrenia and 21 controls with fMRI. Participants with familial risk were recruited from a referral network including community-based health providers and the UNC PRIME (Prevention through Risk Identification, Management and Education) research clinic. Participants with familial risk were recruited based only on having an affected first-degree relative, and not based on presence of clinical symptoms. No participants with familial risk were seeking treatment and no participants were related to one another. Controls were recruited from local schools and the general community. Analyses of demographic data were carried out using ANOVA for age and years of education and using chi-square tests for gender, ethnicity, and handedness. The groups did not significantly differ according to any of these demographic variables (Table 1). Ethnicity was classified by participant self-report at the time of screening by options defined by the investigators in order to match between groups.

Familial risk was defined as having a first-degree family member (sibling or parent) with a diagnosis of schizophrenia or schizoaffective disorder, as determined by the Family Interview for Genetics conducted with the participant's parent (Maxwell, 1992). Diagnosis of the affected relative was confirmed by the Structured Interview for DSM IV Disorders for adults and the Washington University Kiddie Schedule for Affective Disorders and 
Schizophrenia for children (Orvaschel et al., 1982). Of the 21 subjects with familial risk, two had an affected parent and sibling, two had an affected parent only, and 17 had an affected sibling. Seven subjects had a relative with schizoaffective disorder, and fourteen subjects had a relative with schizophrenia.

Exclusion criteria for the familial risk group included presence of a past or current DSM-IV Axis I psychotic or bipolar affective disorder. Because familial risk for schizophrenia is associated with a high likelihood of premorbid disorders (Keshavan et al., 2008), we chose not to exclude high-risk individuals with other Axis I disorders. In our sample, eight participants with familial risk had other diagnoses including attention-deficit hyperactivity disorder (ADHD), learning disorder, anxiety disorder - not otherwise specified, encopresis and enuresis. Within this group, there were three participants who were taking medications for ADHD, including atomoxetine, buproprion and fluoxetine. Other exclusion criteria for the familial risk group included central nervous system disorder (e.g., seizure disorder) or mental retardation (IQ less than 65), current treatment with an antipsychotic medication, and past history of over 12 weeks lifetime cumulative treatment with an antipsychotic. Two participants with familial risk had taken antipsychotic medications in the past, but were not excluded based on our criteria of 12 weeks of lifetime cumulative treatment. Exclusion criteria for the control group included history of a DSM-IV Axis I psychiatric disorder, any psychiatric disorder in a first-degree relative, neurological disorder, and substance abuse disorder. Additional exclusion criteria related to MRI safety included presence of metal in the body and pregnancy. After complete description of the study to the subjects, written informed assent (or consent, if age 18) was obtained, with parents providing written consent as approved by the University of North Carolina at Chapel Hill and Duke University School of Medicine Institutional Review Boards.

The presence of positive, negative, and general prodromal symptoms was assessed for all participants using the Schedule of Prodromal Symptoms (SOPS). The data from the SOPS were collected following inclusion in the study, and were not used as criteria for group selection. The familial risk group showed significantly more symptoms than the control group in positive, negative and disorganized symptoms (Table 1). Younger age in the familial risk group was associated with higher scores on the disorganized symptom scale ( $\mathrm{r}$ $=-0.55, p=0.009)$. There were no other significant relationships found between age and prodromal symptom scores for either group. Additionally, we tested for group differences using an estimate of IQ that was generated from a series of neurocognitive measures for fluency, set shifting, inhibition, working memory, attention, and processing speed (SA1 in supplementary material). No significant differences were found between the groups overall, but the group with familial risk had significantly lower scores in the working memory domain than controls $(F(1,39)=5.93, p=0.02)$.

\subsection{Imaging Task}

Participants performed a visual oddball task requiring the identification of an infrequent target stimulus (a circle), presented among a series of frequent standard stimuli consisting of scrambled images. The task was presented in 8 functional runs, each lasting approximately 4 minutes. Targets made up approximately $4 \%$ of the total trials, with 40 target trials across the experiment. Additionally, there were other task-irrelevant infrequent pictures presented with negative emotional (aversive) or neutral content, each of which accounted for another $4 \%$ of the total trials ( 40 trials each across the experiment). A schematic of the task is presented in the online-only material (SF1). Aversive and neutral pictures were chosen from the International Affective Picture System database, which consists of complex pictures with standardized ratings from adults for arousal and valence (Lang et al., 2005). These pictures have standardized ratings on a scale of $1-9$, with higher numbers reflecting more positive valence and higher arousal. The aversive stimuli consisted of unpleasant or frightening 
pictures that were selected to be age-appropriate for the children and adolescents in the study (e.g., animals). The average valence and arousal ratings for the task-irrelevant aversive stimuli were $3.38(S D=1.78)$ and $6.14(S D=2.08)$, respectively. Average valence and arousal ratings for the neutral stimuli were $6.21(S D=0.26)$ and $3.72(S D=2.15)$. All images were pseudo-randomized, with at least 15 seconds between two targets and/or two aversive/neutral stimuli. Participants pressed one button when the target was presented, and a different button to all other stimuli. Each image was presented for $1500 \mathrm{msec}$, with 500 msec inter-stimulus intervals. Prior to fMRI scanning, participants performed a mock MRI scan and practice run to familiarize them with the MRI environment and task instructions. Additionally, following fMRI scanning, a subset of participants ( 15 controls and 13 with familial high risk) provided subjective ratings of arousal and valence for the images used in the study. The high-risk group rated the aversive pictures as significantly less arousing $(F(1,27)=6.52 ; p=0.02)$ than controls, but there were no group differences in valence ratings.

\subsection{Image Acquisition}

Participants underwent fMRI scanning on a General Electric 3.0 Tesla MRI scanner using a functional gradient echo echo-planar imaging sequence allowing for full-brain coverage (TR: $2000 \mathrm{msec}$; TE: $27 \mathrm{msec}$; FOV: $24 \mathrm{~cm}$; image matrix: $64 \times 64$; Flip Angle: 60; voxel size: $3.75 \times 3.75 \times 3.8 \mathrm{~mm}$; 34 axial slices). Functional runs consisted of 120 time points. Preceding the functional image acquisition, structural MRIs were acquired to obtain 3D coplanar anatomical T1 images using a spoiled gradient-recalled acquisition pulse sequence (TR: $5.16 \mathrm{msec}$; TE: $2.04 \mathrm{msec}$; FOV: $24 \mathrm{~cm}$; image matrix: $256 \times 256$; Flip Angle: 20; voxel size: $0.94 \times 0.94 \times 1.9 \mathrm{~mm} ; 68$ axial slices).

\subsection{Analyses}

2.4.1. Preprocessing-Functional data analyses were carried out using FSL version 4.0 (Oxford Centre for Functional Magnetic Resonance Imaging of the Brain (FMRIB), Oxford University, U.K.) (Smith et al., 2004). Image preprocessing (first-level analysis) steps included using the Brain Extraction Tool (BET) to remove non-brain structures (Smith, 2002), motion correction (Jenkinson et al., 2002), spatial filtering using a Gaussian kernel of full width half maximum $5 \mathrm{~mm}$, high-pass temporal filtering (Jenkinson et al., 2002; Smith et al., 2004), and time-slice correction. No participant had greater than $3 \mathrm{~mm}$ head motion in the $\mathrm{X}, \mathrm{Y}$, or $\mathrm{Z}$ directions. The functional images were co-registered to the structural images in their native space, and the images were then normalized to the Montreal Neurologic Institute standard brain. All registrations were carried out using FMRIB's Linear Image Registration Tool (FLIRT) (Jenkinson and Smith, 2001) using the default adult template. Pre-whitening was carried out with FMRIB's Improved Linear Model (FILM) in order to estimate and account for each voxel's time series autocorrelation (Woolrich et al., 2001).

2.4.2. Voxel-wise Analyses-In order to test which regions were associated with successful executive and emotional processing, we excluded error trials from the analyses. Onset times of events were used to model regressors for each experimental condition, which were convolved with a gamma function to model the hemodynamic response. Whole-brain images of the parameter estimates and variance were generated for each subject for targets and task-irrelevant aversive and neutral stimuli. Contrast images were then generated to create $z$-statistic activation maps where the estimates for each condition were non-zero. To specifically measure activation during target processing, the contrasts were modeled to identify activation significantly greater than zero. Contrast maps were also generated to compare the aversive versus neutral conditions, and the familial risk group was compared to the control group for each condition. The average of these contrast maps across subjects was estimated using FMRIB's Local Analysis of Mixed Effects (FLAME) (Beckmann et al., 
2003; Woolrich et al., 2004) within the fMRI Expert Analysis Tool (FEAT). Group-level activation maps were thresholded using a $Z$-statistic to define contiguous clusters of activation. Anatomical regions of activation were identified according to the probabilistic Harvard-Oxford Cortical and Subcortical Atlases within FSLView.

Additionally, whole-brain voxelwise correlation analyses were run with age as a covariate to determine which regions showed a significant positive and negative relationship with age for both targets and the aversive versus neutral contrast. Significant clusters for all analyses were corrected for multiple comparisons using a false discovery rate threshold of $p<0.05$. The false discovery rate correction was applied to all voxels in the brain in order to threshold those voxels reaching significance at the corrected p-value of 0.05 , as described by Genovese et al. (2002). Significant clusters of activation were identified by having a minimum cluster size of 8 contiguous voxels that reached the FDR-corrected threshold (as in Dichter et al., 2010). Finally, in order to directly test whether the regions that showed group differences were explained by age effects, activity within all voxels was extracted from those regions, defined anatomically by the Harvard-Oxford Cortical and Subcortical Structural Atlases within FSLView. ANOVAs were run on the extracted parameter estimates to determine whether age significantly correlated with activation in these areas. We also included an analysis with age as a nuisance variable to determine whether age contributed to the group differences. Additionally, because three high-risk participants were taking medication for ADHD, we performed an analysis with medication status as a covariate to determine whether it affected the group differences.

Additionally, in order to account for whether functional differences between groups might be due to differences in spatial normalization, we performed an analysis on the degree of voxel warping between groups. Details are presented in the supplementary appendix (SA2). No group differences were detected.

\section{Results}

\subsection{Behavioral}

No significant differences were found between the two groups on performance measures for the target detection task. However, the familial risk group showed poorer accuracy during identification of non-target stimuli overall $(F(1,40)=5.97, p=0.02)$, but no group by emotion interaction was found. More detailed supplementary behavioral findings are presented in the online-only results section (SA3), including the relationship between performance and activation measures (SA4).

\subsection{Brain Activity during Target and Aversive Conditions}

Significant within-group activation clusters are presented in the online-only material (ST1, ST2). During targets, both groups activated clusters within prefrontal, anterior caudate, insular, and posterior parietal areas. During aversive trials, both groups showed activation in the right amygdala, bilateral orbitofrontal cortex, fusiform cortex and visual cortical areas. During aversive versus neutral trials, both groups activated inferior frontal and parietal cortical areas, with controls showing additional activation in inferior temporal and fusiform cortex.

The majority of areas showing group differences were within areas significantly activated by the task, although a few regions outside of the task-related network also showed group differences (noted on tables). Between-group analyses indicated that during target processing, the familial risk group showed significant hyperactivation relative to controls in fronto-striate circuitry. Voxel-based analyses indicated significant hyperactivation in areas within the inferior frontal gyrus, caudate, and inferior temporal gyrus in the familial risk 
group relative to controls (Table 2, Figure 1A). In contrast, areas where controls activated more than the familial risk group during target processing included clusters in the middle frontal gyrus, insula, sensorimotor cortices, occipital cortex, and temporal cortical regions (Table 2, Figure 1B). Additional covariate analyses with medication status for ADHD revealed no changes in the group difference activation patterns. During processing of taskirrelevant aversive relative to neutral pictures, the familial risk group showed hyperactivation in only a cluster within the central opercular cortex (Table 2, Figure 1C), while controls showed greater activation than the familial risk group in areas within the anterior cingulate cortex and precuneus (Table 2, Figure 1D). However, only the anterior cingulate cortex reached significance when controlling for ADHD medication status.

\subsection{Age-Dependent Developmental Changes in Brain Activity}

Whole-brain voxelwise correlation analyses revealed that the familial risk group showed differences in age-related activation patterns during both target and emotional processing relative to controls. A significant group by age interaction effect during target processing was found across a network of regions, with the predominant pattern reflecting a stronger positive age correlation in the familial risk group compared to controls in the anterior cingulate, medial frontal cortex, insula, thalamus, and temporal and occipital cortex (Table 3 , Figure 2A, SF2). Correlation analyses within each group indicated that the familial risk group showed a significant positive age correlation in several of these areas, including clusters in the thalamus, temporal and occipital cortex, while activation in the controls showed a significant negative age relationship in areas within the anterior cingulate gyrus and insula (ST3). In other words, the older adolescents in the familial risk group tended to show increased recruitment of these regions during target processing, while controls showed relatively decreased recruitment with age.

During the aversive versus neutral contrast, a different pattern indicated that controls had a stronger positive age relationship than the familial risk group across multiple regions, including clusters in the inferior frontal cortex, frontal pole, precentral and postcentral gyri, and occipital cortex (Table 3, Figure 2B). Comparison of the within-group correlations showed that these interactions primarily reflected age-related decreases in activation in the familial risk group, while fewer areas in the controls showed a significant age relationship during the aversive versus neutral contrast (ST3). The older adolescents in the familial risk group, therefore, tended to show less activation to aversive versus neutral pictures, while activity in controls was relatively similar across different ages.

Finally, within areas that showed significant group differences in activation to targets and the aversive versus neutral contrasts, no significant group by age interactions were detected in any region, suggesting that the differential recruitment of regions with age did not directly account for the overall group differences in activation patterns. Furthermore, additional analyses with age as a nuisance regressor revealed no changes in the group difference activation patterns.

\section{Discussion}

The results of the current study indicated that during selective attention processes, individuals with familial high risk for developing schizophrenia showed hyperactivation relative to control subjects in fronto-striate regions including the left inferior prefrontal cortex and bilateral caudate. Additionally, the familial risk group showed relative hypoactivation relative to controls within the middle frontal gyrus, insula, and thalamus. Correlations with age revealed differential engagement of insula, thalamus, temporal and occipital areas between groups, with the familial risk group showing relatively enhanced activation with increased age compared to controls. During processing of task-irrelevant 
aversive pictures relative to neutral pictures, the familial risk group showed decreased activation of the anterior cingulate cortex, with relatively decreased age-related engagement of inferior prefrontal and occipital regions. The results overall suggest that changes in neural recruitment in fronto-striate, insular, and cingulate areas during selective attention and emotional processing may reflect a familial-mediated endophenotype, potentially indicating changes in allocation of cognitive control for task-relevant and irrelevant stimuli.

Additionally, age-related changes in recruitment of frontal, insular, thalamic, and other temporal and occipital regions may reflect developmental changes in the function of these circuits in individuals at risk for developing schizophrenia.

Patterns of prefrontal hyperactivation have been observed in many studies of patients with schizophrenia and adults with affected relatives (Manoach et al., 1999; Whalley et al., 2005a). These findings have been linked to a model of cortical inefficiency, which posits that because of aberrant connectivity in schizophrenia, the networks activated during cognitive processing do so in an inefficient manner (Manoach et al., 1999). In contrast, prefrontal hypoactivation has been found to be associated with engagement in more difficult cognitive tasks, when the task complexity exceeds the individual's capacity (Manoach, 2003). Consistent with the current study's findings, Jacobsen and colleagues (2010) have also shown hypoactivation in the dorsolateral prefrontal cortex during cognitive control in children with familial risk, suggesting the possibility that younger at-risk populations may have a decreased capacity to process attentional demands.

The current study's findings of hyperactivation in the caudate in the familial risk group are consistent with multiple studies highlighting changes in this region as potentially reflecting an early risk marker for schizophrenia (Rajarethinam et al., 2007). Increased engagement of the caudate was also identified as a significant predictor of development of psychosis in adolescents with familial risk (Sabb et al., 2010). Several studies examining volumetric changes in the caudate have shown inconsistent results (Levitt et al., 2010), which may be due to confounding effects of antipsychotic medication effects on caudate volume (Keshavan et al., 1994). However, studies with medication-naïve participants have demonstrated reduced volumes in adults with schizophrenia and their relatives (Keshavan et al., 1998; Rajarethinam et al., 2007), suggesting that it may be a trait-related marker for schizophrenia.

During emotional processing, the familial risk group was found to show relative hypoactivation in the anterior cingulate cortex during processing of task-irrelevant aversive pictures relative to neutral pictures. The anterior cingulate has been found to show reduced activation during cognitive control in children at risk for developing schizophrenia (Jacobson et al., 2010), with structural changes found to be associated with increased risk of future development of psychosis (Pantelis et al., 2003). It may be that in younger at-risk adolescents, decreased activity in the anterior cingulate could reflect early alterations in these regulatory mechanisms that may subsequently change in adulthood. This hypoactivation may reflect decreased attention allocated to aversive stimuli, as the group with familial risk rated the pictures as less arousing than controls.

The familial risk group in the current study also showed differential increased recruitment of multiple additional regions with age, including the anterior cingulate, thalamus, temporal and occipital areas. These patterns of hyperactivation suggest a potential compensatory function, where increased response selection and inhibition is required to process cognitively demanding stimuli. While the data in the current study showed that both groups had decreasing dorsolateral prefrontal activity with age, controls also showed decreased agerelated activity across a multitude of other cortical areas while the high-risk group tended to show age-related hyperactivation. This suggests the possibility that the prefrontal cortex 
functional trajectory may reflect an exaggerated variation of the normal pattern with overall decreased activity compared to controls, while the pattern of development of hyperactivation across other cortical regions may differ in high-risk individuals.

Furthermore, the network of regions showing greater age-related hyperactivation in the familial risk group was similarly found to be altered in a study by Bhojraj and colleagues (2011), which found age-related volumetric loss in at-risk adolescents. This volumetric loss was associated with increased prodromal symptoms at subsequent follow-up. This agerelated volume loss may reflect heritable neurodevelopmental changes associated with excessive synaptic pruning in the peri-adolescent period of development (Hayashi-Takagi and Sawa, 2010). Future studies may address whether progressive volume loss in these circuits is directly associated with greater compensatory activity and cognitive decline. Longitudinal data will be necessary to further clarify how the age-related hyperactivation may relate to the normal developmental trajectory of activation patterns.

There are several points of caution that should be taken into account when interpreting the results of the current study. One limitation is that our task did not elicit significant activation of the amygdala during the aversive minus neutral condition, which could provide additional information about emotional processing changes in the familial risk group. The finding of significant amygdala activation during the aversive condition, but not aversive minus neutral, likely reflects the nature of the stimuli that were selected to be appropriate for children and adolescents. It is also important to note that the majority of the individuals with familial high risk will not develop schizophrenia (Parnas et al., 1993), as the risk for developing schizophrenia is estimated to be about 10 percent in individuals with first-degree affected family members (Barondes, 1999). Our results therefore reflect biological phenomena and phenotypes that may be associated with familial risk, and do not necessarily reflect heritability or prediction of future development of the disease. Finally, our analyses of age effects were limited by the sample size and cross-sectional nature of our current study, and future longitudinal investigations of age effects in high-risk individuals may be able to more directly characterize the developmental trajectories of functional changes in high-risk adolescents.

We also note that the heterogeneity of our high-risk sample with regard to other diagnoses could influence the patterns of group differences. While our sample of high-risk participants did not include individuals with psychosis, we chose not to exclude individuals with other Axis-I psychiatric disorders. High-risk individuals have been found to have higher frequencies of non-psychotic diagnoses, including anxiety and depression (Amminger et al., 2000) and "externalizing" disorders, including ADHD (Keshavan et al., 2003). Keshavan and colleagues (2008) have suggested that externalizing disorders in particular may represent pre-psychotic symptoms in a subgroup with higher risk for developing schizophrenia. Therefore, the presence of other Axis-I diagnoses may be an important manifestation of the high-risk phenotype with greater power to predict psychosis. We note that a limitation related to inclusion of individuals with other diagnoses is that the medications taken by the three individuals with ADHD may influence the group difference patterns. However, controlling for ADHD status in our sample led to minimal changes in the group differences, suggesting that the primary findings are likely not due to medication effects.

In summary, our findings indicate that altered activation in circuitry supporting executive and emotional processing is associated with familial risk for schizophrenia during childhood and adolescence, prior to the potential onset of the disorder. Although these findings do not predict disease development, they demonstrate that functional changes in vulnerable neural circuits can be found in at-risk individuals at an early age, potentially suggesting a heritable 
change in neurodevelopment. Changes in fronto-striate, insular, and anterior cingulate regions, in combination with age-dependent changes in neural recruitment, may reflect patterns of neural inefficiency that develop early in adolescence. It will be critical to understand in the future how specific genetic mechanisms and their interaction with neurodevelopmental factors influence these patterns, and whether these neural correlates might be used to develop strategies for prevention and treatment.

\section{Supplementary Material}

Refer to Web version on PubMed Central for supplementary material.

\section{Acknowledgments}

We thank Erin Douglas, Anna Evans, and Carolyn Bellion for their contributions to participant recruitment and clinical assessment. We also thank Michael Casp, Zoe Englander, Justin Woodlief, and James Carter for their contributions to data collection and analysis, and Robert M. Hamer for consultation on statistical analysis and editing of the manuscript. Finally, we thank the individuals and their families who participated in this study.

This study was supported by Conte center grant P50 MH064065 from the National Institute of Mental Health. Dr. Hart was supported by T32 HD040127 from the National Institute of Child Health and Human Development.

\section{References}

Amminger GP, Pape S, Rock D, Roberts SA, Squires-Wheeler E, Kestenbaum C, Erlenmeyer-Kimling L. The New York High-Risk Project: comorbidity for axis I disorders is preceded by childhood behavioral disturbance. Journal of Nervous and Mental Disease. 2000; 188:751-756. [PubMed: 11093377]

Barondes SH. An agenda for psychiatric genetics. Archives of General Psychiatry. 1999; 56:549-552. [PubMed: 10359469]

Beckmann CF, Jenkinson M, Smith SM. General multilevel linear modeling for group analysis in FMRI. Neuroimage. 2003; 20:1052-1063. [PubMed: 14568475]

Bhojraj TS, Sweeney JA, Prasad KM, Eack SM, Francis AN, Miewald JM, Montrose DM, Keshavan MS. Gray matter loss in young relatives at risk for schizophrenia: relation with prodromal psychopathology. Neuroimage. 2011; 54(Suppl 1):S272-279. [PubMed: 20441795]

Bonner-Jackson A, Csernansky JG, Barch DM. Levels-of-processing effects in first-degree relatives of individuals with schizophrenia. Biological Psychiatry. 2007; 61:1141-1147. [PubMed: 17123479]

Bramon E, McDonald C, Croft RJ, Landau S, Filbey F, Gruzelier JH, Sham PC, Frangou S, Murray RM. Is the P300 wave an endophenotype for schizophrenia? A meta-analysis and a family study. Neuroimage. 2005; 27:960-968. [PubMed: 16009570]

Cornblatt BA, Keilp JG. Impaired attention, genetics, and the pathophysiology of schizophrenia [published erratum appears in Schizophr Bull 1994;20(2):248]. Schizophrenia Bulletin. 1994; 20:31-46. [PubMed: 8197420]

Dichter GS, Bellion C, Casp M, Belger A. Impaired modulation of attention and emotion in schizophrenia. Schizophrenia Bulletin. 2010; 36:595-606. [PubMed: 18843096]

Diwadkar VA, Wadehra S, Pruitt P, Keshavan MS, Rajan U, Zajac-Benitez C, Eickhoff SB. Disordered corticolimbic interactions during affective processing in children and adolescents at risk for schizophrenia revealed by functional magnetic resonance imaging and dynamic causal modeling. Archives of General Psychiatry. 2012; 69:231-242. [PubMed: 22393216]

Faraone, SV.; Tsuang, D.; Tsuang, MT. Genetics of Mental Disorders: A Guide for Students, Clinicians, and Researchers. Guilford; New York: 1999.

Feinberg I. Schizophrenia: caused by a fault in programmed synaptic elimination during adolescence? Journal of Psychiatric Research. 1982; 17:319-334. [PubMed: 7187776]

Genovese CR, Lazar NA, Nichols T. Thresholding of statistical maps in functional neuroimaging using the false discovery rate. Neuroimage. 2002; 15:870-878. [PubMed: 11906227] 
Glatt SJ, Stone WS, Faraone SV, Seidman LJ, Tsuang MT. Psychopathology, personality traits and social development of young first-degree relatives of patients with schizophrenia. British Journal of Psychiatry. 2006; 189:337-345. [PubMed: 17012657]

Gogtay N. Cortical brain development in schizophrenia: insights from neuroimaging studies in childhood-onset schizophrenia. Schizophrenia Bulletin. 2008; 34:30-36. [PubMed: 17906336]

Gogtay N, Sporn A, Clasen LS, Greenstein D, Giedd JN, Lenane M, Gochman PA, Zijdenbos A, Rapoport JL. Structural brain MRI abnormalities in healthy siblings of patients with childhoodonset schizophrenia. American Journal of Psychiatry. 2003; 160:569-571. [PubMed: 12611841]

Gottesman II, Gould TD. The endophenotype concept in psychiatry: etymology and strategic intentions. American Journal of Psychiatry. 2003; 160:636-645. [PubMed: 12668349]

Gur RE, Calkins ME, Gur RC, Horan WP, Nuechterlein KH, Seidman LJ, Stone WS. The Consortium on the Genetics of Schizophrenia: neurocognitive endophenotypes. Schizophrenia Bulletin. 2007a; 33:49-68. [PubMed: 17101692]

Gur RE, Nimgaonkar VL, Almasy L, Calkins ME, Ragland JD, Pogue-Geile MF, Kanes S, Blangero J, Gur RC. Neurocognitive endophenotypes in a multiplex multigenerational family study of schizophrenia. American Journal of Psychiatry. 2007b; 164:813-819. [PubMed: 17475741]

Hare TA, Tottenham N, Galvan A, Voss HU, Glover GH, Casey BJ. Biological substrates of emotional reactivity and regulation in adolescence during an emotional go-nogo task. Biological Psychiatry. 2008; 63:927-934. [PubMed: 18452757]

Hayashi-Takagi A, Sawa A. Disturbed synaptic connectivity in schizophrenia: convergence of genetic risk factors during neurodevelopment. Brain Research Bulletin. 2010; 83:140-146. [PubMed: 20433911]

Jacobson S, Kelleher I, Harley M, Murtagh A, Clarke M, Blanchard M, Connolly C, O'Hanlon E, Garavan H, Cannon M. Structural and functional brain correlates of subclinical psychotic symptoms in 11-13 year old schoolchildren. Neuroimage. 2010; 49:1875-1885. [PubMed: 19770054]

Jenkinson M, Smith S. A global optimisation method for robust affine registration of brain images. Medical Image Analysis. 2001; 5:143-156. [PubMed: 11516708]

Jenkinson M, Bannister P, Brady M, Smith S. Improved optimization for the robust and accurate linear registration and motion correction of brain images. Neuroimage. 2002; 17:825-841. [PubMed: 12377157]

Keshavan M, Montrose DM, Rajarethinam R, Diwadkar V, Prasad K, Sweeney JA. Psychopathology among offspring of parents with schizophrenia: relationship to premorbid impairments. Schizophrenia Research. 2008; 103:114-120. [PubMed: 18442896]

Keshavan MS, Rosenberg D, Sweeney JA, Pettegrew JW. Decreased caudate volume in neurolepticnaive psychotic patients. American Journal of Psychiatry. 1998; 155:774-778. [PubMed: 9619149]

Keshavan MS, Sujata M, Mehra A, Montrose DM, Sweeney JA. Psychosis proneness and ADHD in young relatives of schizophrenia patients. Schizophrenia Research. 2003; 59:85-92. [PubMed: 12413647]

Keshavan MS, Bagwell WW, Haas GL, Sweeney JA, Schooler NR, Pettegrew JW. Changes in caudate volume with neuroleptic treatment. Lancet. 1994; 344:1434. [PubMed: 7968091]

Keshavan MS, Dick E, Mankowski I, Harenski K, Montrose DM, Diwadkar V, DeBellis M. Decreased left amygdala and hippocampal volumes in young offspring at risk for schizophrenia. Schizophrenia Research. 2002; 58:173-183. [PubMed: 12409156]

Keshavan MS, Kulkarni S, Bhojraj T, Francis A, Diwadkar V, Montrose DM, Seidman LJ, Sweeney J. Premorbid cognitive deficits in young relatives of schizophrenia patients. Frontiers in Human Neuroscience. 2010; 3:62. [PubMed: 20300465]

Kety SS, Rosenthal D, Wender PH, Schulsinger F. Mental illness in the biological and adoptive families of adpoted schizophrenics. American Journal of Psychiatry. 1971; 128:302-306. [PubMed: 5570994]

Kiehl KA, Liddle PF. An event-related functional magnetic resonance imaging study of an auditory oddball task in schizophrenia. Schizophrenia Research. 2001; 48:159-171. [PubMed: 11295369] 
Lang, PJ.; Bradley, MM.; Cuthbert, BN. Technical Report A-6. The Center for Research in Psychophysiology, University of Florida; Gainesville, FL: 2005. International affective picture system (IAPS): Digitized photographs, instruction manual and affective ratings.

Levitt, JJ.; Bobrow, L.; Lucia, D.; Srinivasan, P. A Selective Review of Volumetric and Morphometric Imaging in Schizophrenia. In: Swerdlow, NR., editor. Behavioral Neurobiology of Schizophrenia and Its Treatment. Springer-Verlag; New York: 2010. p. 243-282.

Luna B, Padmanabhan A, O'Hearn K. What has fMRI told us about the development of cognitive control through adolescence? Brain and Cognition. 2010; 72:101-113. [PubMed: 19765880]

Luna B, Thulborn KR, Munoz DP, Merriam EP, Garver KE, Minshew NJ, Keshavan MS, Genovese CR, Eddy WF, Sweeney JA. Maturation of widely distributed brain function subserves cognitive development. Neuroimage. 2001; 13:786-793. [PubMed: 11304075]

Manoach DS. Prefrontal cortex dysfunction during working memory performance in schizophrenia: reconciling discrepant findings. Schizophrenia Research. 2003; 60:285-298. [PubMed: 12591590]

Manoach DS, Press DZ, Thangaraj V, Searl MM, Goff DC, Halpern E, Saper CB, Warach S. Schizophrenic subjects activate dorsolateral prefrontal cortex during a working memory task, as measured by fMRI. Biological Psychiatry. 1999; 45:1128-1137. [PubMed: 10331104]

Maxwell, ME. Manual for the FIGS (Family Interview for Genetic Studies). Bethesda, MD: Clinical Neurogenetics Branch, Intramural research Program, National Institute of Mental Health; 1992.

Meyer-Lindenberg A, Weinberger DR. Intermediate phenotypes and genetic mechanisms of psychiatric disorders. Nat Rev Neurosci. 2006; 7:818-827. [PubMed: 16988657]

Meyer-Lindenberg A, Miletich RS, Kohn PD, Esposito G, Carson RE, Quarantelli M, Weinberger DR, Berman KF. Reduced prefrontal activity predicts exaggerated striatal dopaminergic function in schizophrenia. Nature Neuroscience. 2002; 5:267-271.

Nuechterlein KH, Dawson ME. Information processing and attentional functioning in the developmental course of schizophrenic disorders. Schizophrenia Bulletin. 1984; 10:160-203. [PubMed: 6729409]

Nuechterlein KH, Dawson ME, Ventura J, Gitlin M, Subotnik KL, Snyder KS, Mintz J, Bartzokis G. The vulnerability/stress model of schizophrenic relapse: a longitudinal study. Acta Psychiatrica Scandinavica, Supplementum. 1994; 382:58-64. [PubMed: 8091999]

Orvaschel H, Puig-Antich J, Chambers W, Tabrizi MA, Johnson R. Retrospective assessment of prepubertal major depression with the Kiddie-SADS-e. Journal of the American Academy of Child and Adolescent Psychiatry. 1982; 21:392-397.

Pantelis C, Velakoulis D, McGorry PD, Wood SJ, Suckling J, Phillips LJ, Yung AR, Bullmore ET, Brewer W, Soulsby B, Desmond P, McGuire PK. Neuroanatomical abnormalities before and after onset of psychosis: a cross-sectional and longitudinal MRI comparison. Lancet. 2003; 361:281288. [PubMed: 12559861]

Parnas J, Cannon TD, Jacobsen B, Schulsinger H, Schulsinger F, Mednick SA. Lifetime DSM-III-R diagnostic outcomes in the offspring of schizophrenic mothers. Results from the Copenhagen High-Risk Study. Archives of General Psychiatry. 1993; 50:707-714. [PubMed: 8357296]

Pearlson GD, Calhoun VD. Convergent approaches for defining functional imaging endophenotypes in schizophrenia. Frontiers in Human Neuroscience. 2009; 3:37. [PubMed: 19956400]

Rajarethinam R, Upadhyaya A, Tsou P, Upadhyaya M, Keshavan MS. Caudate volume in offspring of patients with schizophrenia. British Journal of Psychiatry. 2007; 191:258-259. [PubMed: 17766768]

Rubia K, Smith AB, Woolley J, Nosarti C, Heyman I, Taylor E, Brammer M. Progressive increase of frontostriatal brain activation from childhood to adulthood during event-related tasks of cognitive control. Human Brain Mapping. 2006; 27:973-993. [PubMed: 16683265]

Sabb FW, van Erp TG, Hardt ME, Dapretto M, Caplan R, Cannon TD, Bearden CE. Language network dysfunction as a predictor of outcome in youth at clinical high risk for psychosis. Schizophrenia Research. 2010; 116:173-183. [PubMed: 19861234]

Smith SM. Fast robust automated brain extraction. Human Brain Mapping. 2002; 17:143-155. [PubMed: 12391568]

Smith SM, Jenkinson M, Woolrich MW, Beckmann CF, Behrens TE, Johansen-Berg H, Bannister PR, De Luca M, Drobnjak I, Flitney DE, Niazy RK, Saunders J, Vickers J, Zhang Y, De Stefano N, 
Brady JM, Matthews PM. Advances in functional and structural MR image analysis and implementation as FSL. Neuroimage. 2004; 23(Suppl 1):S208-219. [PubMed: 15501092]

Tian L, Meng C, Yan H, Zhao Q, Liu Q, Yan J, Han Y, Yuan H, Wang L, Yue W, Zhang Y, Li X, Zhu $\mathrm{C}, \mathrm{He}$ Y, Zhang D. Convergent evidence from multimodal imaging reveals amygdala abnormalities in schizophrenic patients and their first-degree relatives. PLoS One. 2011; 6:e28794. [PubMed: 22174900]

Vink M, Ramsey NF, Raemaekers M, Kahn RS. Striatal dysfunction in schizophrenia and unaffected relatives. Biological Psychiatry. 2006; 60:32-39. [PubMed: 16603134]

Weinberger DR. Implications of normal brain development for the pathogenesis of schizophrenia. Archives of General Psychiatry. 1987; 44:660-669. [PubMed: 3606332]

Whalley HC, Whyte MC, Johnstone EC, Lawrie SM. Neural correlates of enhanced genetic risk for schizophrenia. Neuroscientist. 2005a; 11:238-249. [PubMed: 15911873]

Whalley HC, Simonotto E, Marshall I, Owens DG, Goddard NH, Johnstone EC, Lawrie SM. Functional disconnectivity in subjects at high genetic risk of schizophrenia. Brain. 2005b; 128:2097-2108. [PubMed: 15930046]

Woolrich MW, Ripley BD, Brady M, Smith SM. Temporal autocorrelation in univariate linear modeling of FMRI data. Neuroimage. 2001; 14:1370-1386. [PubMed: 11707093]

Woolrich MW, Behrens TE, Beckmann CF, Jenkinson M, Smith SM. Multilevel linear modelling for FMRI group analysis using Bayesian inference. Neuroimage. 2004; 21:1732-1747. [PubMed: 15050594] 


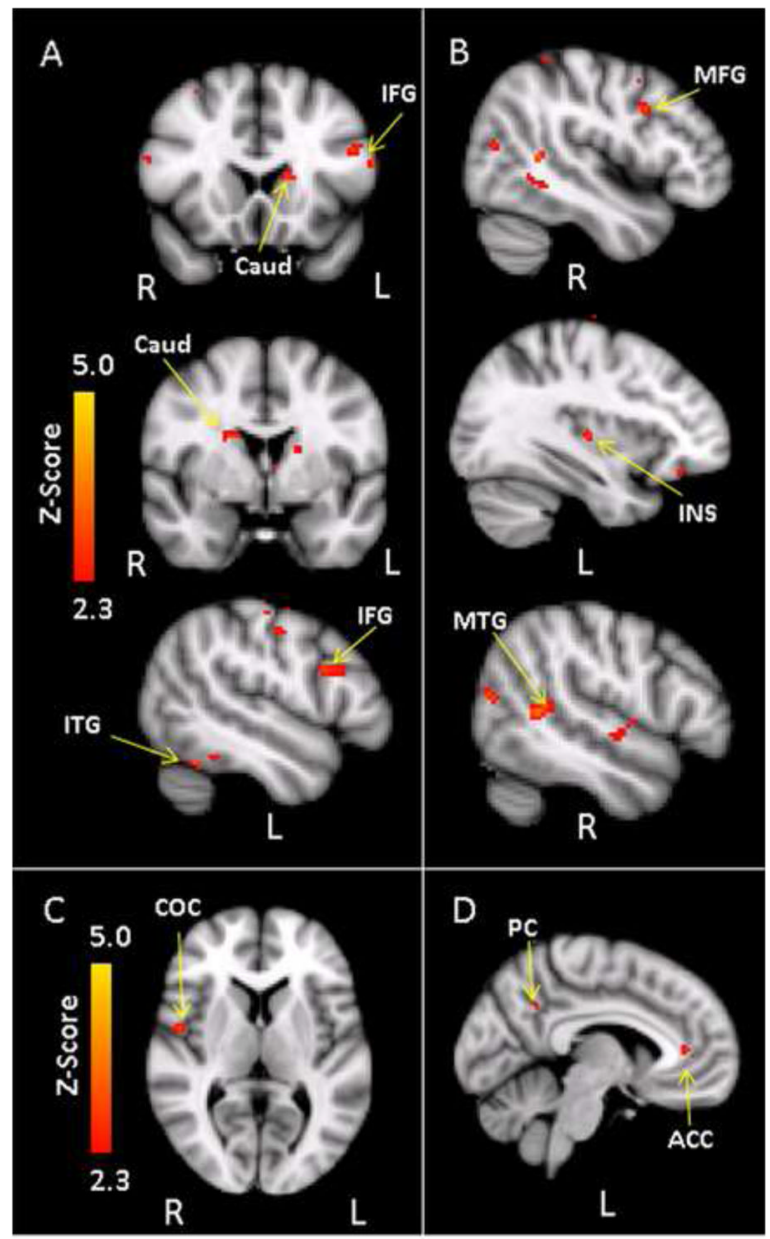

Figure 1.

(A) Areas where the familial high risk group $(n=21)$ showed greater activation than controls $(n=21)$ during target processing. CAUD $=$ Caudate IFG = Inferior frontal gyrus; ITG = Inferior temporal gyrus. (B) Areas where controls showed greater activation than the familial high risk group during target processing. INS $=$ Insula; $\mathrm{MFG}=$ Middle frontal gyrus; $\mathrm{MTG}=$ Middle temporal gyrus. (C) Areas where the familial high risk group activated more than controls during the Aversive $>$ Neutral contrast. $\mathrm{COC}=$ Central opercular cortex. (D) Areas where the controls activated more than the familial high risk group during the Aversive $>$ Neutral contrast. $\mathrm{ACC}=$ Anterior cingulate cortex $\mathrm{PC}=$ Precuneus. 


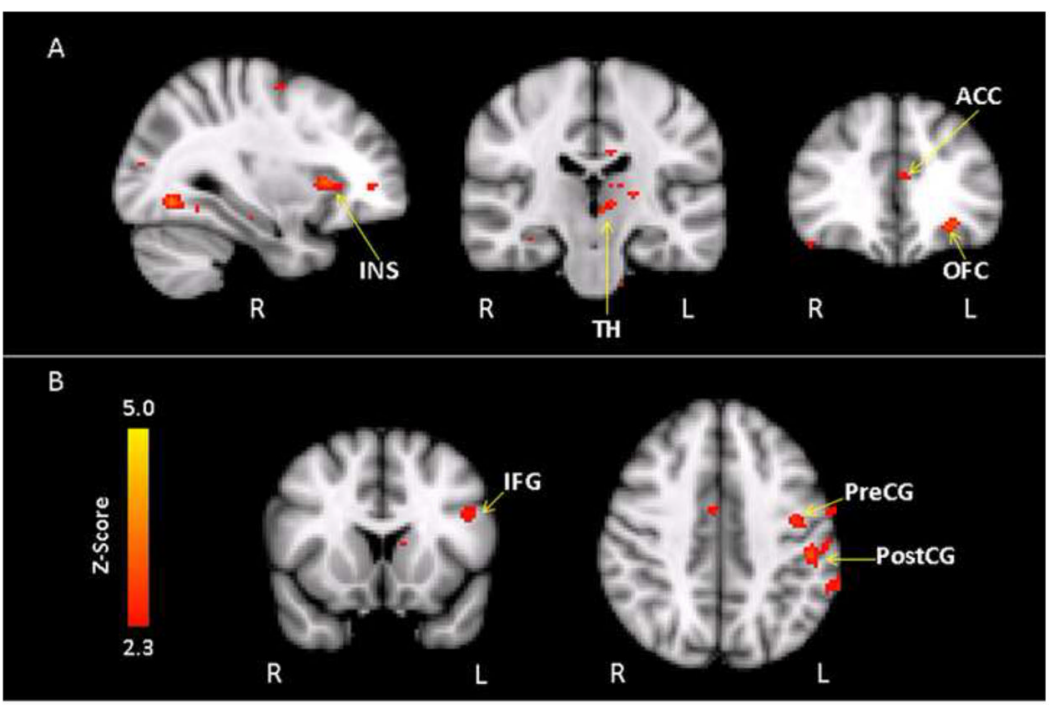

Figure 2.

(A) Areas where the correlation with age was more positive for the familial high risk group than controls during target processing. $\mathrm{ACC}=$ Anterior cingulate cortex; INS = Insula; OFC $=$ Orbitofrontal cortex; $\mathrm{TH}=$ Thalamus. (B) Areas where the correlation with age was more positive for controls than the familial high risk group during the Aversive > Neutral contrast. IFG = Inferior frontal gyrus; PostCG = Postcentral gyrus; PreCG = Precentral gyrus. 
Table 1

Clinical and Demographic Characteristics

\begin{tabular}{lccc}
\hline Characteristic & Controls $(\mathbf{n}=21)$ & Familial High Risk $(\mathbf{n}=\mathbf{2 1})$ & $\boldsymbol{P}$ Value \\
\hline Age, Mean y, SD (Range) & $14.1+/-2.57(9-18)$ & $14.4+/-2.56(9-18)$ & 0.72 \\
Gender, No., \% F & $10(48 \%)$ & $11(52 \%)$ & 0.76 \\
Ethnicity, No., \% White & $18(86 \%)$ & $16(76 \%)$ & 0.7 \\
Education, Mean y, SD (Range) & $7.52+/-2.5(2-11)$ & $7.62+/-2.63(2-11)$ & 0.91 \\
Handedness, No., \% Right & $15(71 \%)$ & $19(90 \%)$ & 0.24 \\
Prodromal symptoms: Positive symptom subscale, Mean, SD & $0.67+/-1.4$ & $2.52+/-2.5$ & $* 0.002$ \\
Prodromal symptoms: Negative symptom subscale, Mean, SD & $0.67+/-1.6$ & $2.9+/-2.7$ & $* 0.002$ \\
Prodromal symptoms: Disorganization symptom subscale, Mean, SD & $0.29+/-0.8$ & $1.19+/-1.4$ & $* 0.02$
\end{tabular}




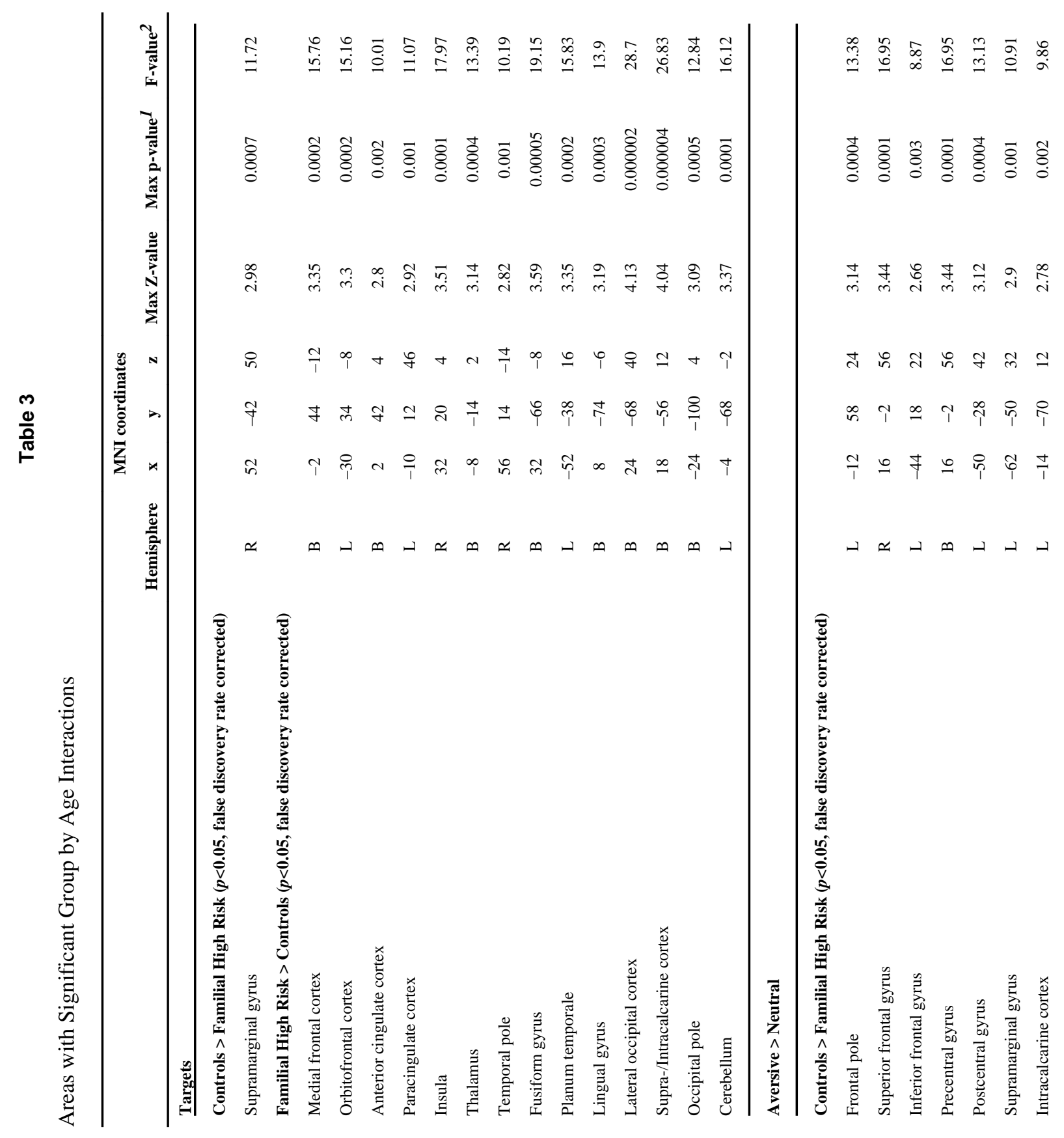




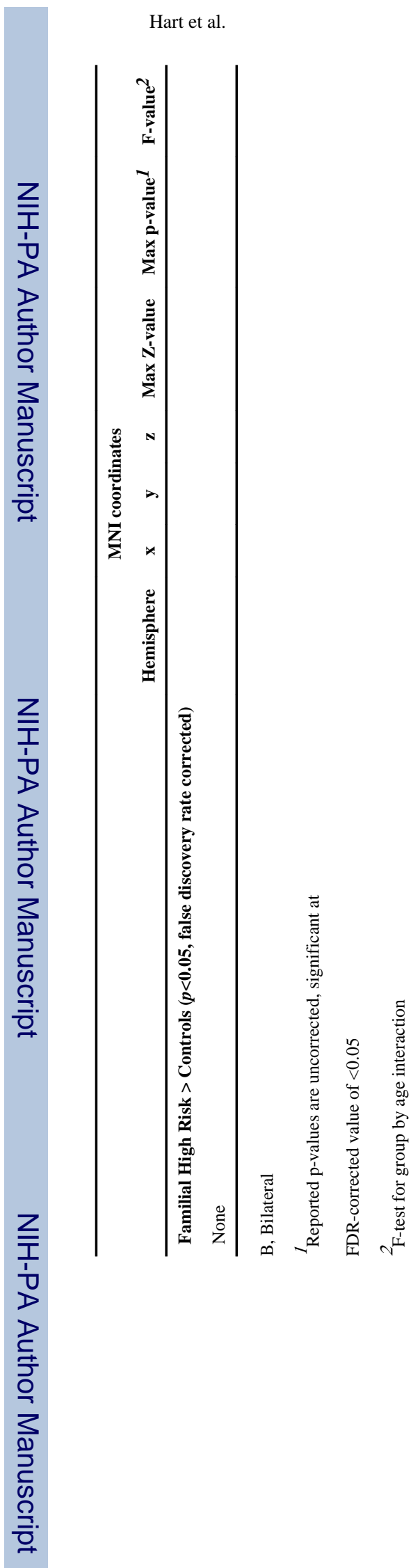

Psychiatry Res. Author manuscript; available in PMC 2014 April 30. 